\title{
Existence of anti-periodic (differentiable) mild solutions to semilinear differential equations with nondense domain
}

\author{
Jinghuai Liu ${ }^{1,2^{*}}$ and Litao Zhang ${ }^{1,2}$
}

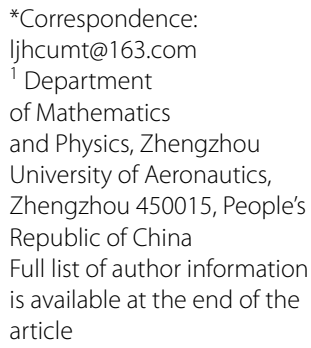

\begin{abstract}
In this paper, we investigate the existence of anti-periodic (or anti-periodic differentiable) mild solutions to the semilinear differential equation $u^{\prime}(t)=A u(t)+f(t, u(t))$ with nondense domain. Furthermore, an example is given to illustrate our results.
\end{abstract}

Keywords: Anti-periodic (differentiable) function, Mild solutions, Hille-Yosida operators, Semilinear differential equations

Mathematics Subject Classification: 35B10, 47D06

\section{Background}

In this paper, we study the existence of anti-periodic (or anti-periodic differentiable) mild solutions to the semilinear differential equation

$$
u^{\prime}(t)=A u(t)+f(t, u(t)), \quad t \in R
$$

where $A$ is an unbounded linear operator, assumed to be Hille-Yosida of negative $\omega$ type having the domain $\mathrm{D}(\mathrm{A})$ not necessarily dense in some Banach spaces $X$ (for more details, see "Preliminaries" section), and $f: R \times X_{0} \rightarrow X$ is a suitable function.

The problem about the existence of anti-periodic solutions constitutes one of the most attractive topics in qualitative theory of differential equations due to its applications in engineering, physics, control theory and other subjects. We refer to the works of Aftabizadeh et al. (1994), Al-Islam et al. (2012), Aizicovici et al. (2001), Cao et al. (2012), Chen et al. (2007), Haraux (1989), Liu et al. (2015), Liu et al. (2015), N'Guérékata and Valmorin (2012), Okochi (1990), Wang and Chen (2013), Yang and Srivastava (2015), Abdurahman and Jiang (2015), Xu (2016), Chadli et al. (2016) and the references therein. However, most of these problems need to be studied in abstract spaces and operators are defined over nondense domain. The literature concerning the existence of anti-periodic solutions for differential equations with nondense domain is new.

To the best of our knowledge, the existence of anti-periodic (or anti-periodic differentiable) mild solutions for semilinear differential equation with nondense domain constitutes until now an untreated original problem. This fact is the main motivations of this 
paper. To illustrate our abstract results, the existence and uniqueness of anti-periodic solutions to a partial differential equation is discussed.

The paper is organized as follows: In "Preliminaries" section, we give some definitions and fix notations which will be used in the sequel. In "Main results and proofs" section, the existence of anti-periodic (or anti-periodic differentiable) mild solution to some semilinear differential equations in Banach space are studied. In "An example" section, an example is given to illustrate our main results.

\section{Preliminaries}

In this section we recall some definitions and fix notations which will be used in the sequel. We assume that $X$ is a Banach space endowed with the norm $\|\cdot\|$ and $B(X)$ stands for the Banach space of all bounded linear operators from $X$ to itself. $R^{+}$denotes the set of nonnegative real numbers. $C_{b}(R, X)$ denotes the space of all bounded continuous functions from $R \rightarrow X$. Moreover, we denote by $C^{1}(R, X)$ the space of all functions $R \rightarrow X$ which have a continuous derivative on $R . C_{b}^{1}(R, X)$ is the subspace of $C^{1}(R, X)$ consists of such functions satisfying

$$
\sup _{t \in R}\left(\|f(t)\|+\left\|f^{\prime}(t)\right\|\right)<\infty
$$

It is clear that $C_{b}^{1}(R, X)$ turns out to be a Banach space with the norm

$$
\|f\|_{C_{b}^{1}(R, X)}=\sup _{t \in R}\left(\|f(t)\|+\left\|f^{\prime}(t)\right\|\right) .
$$

We first recall some properties of Hille-Yosida operators and extrapolation spaces. For more details, we refer to Amir and Maniar (1999), Agarwal et al. (2011), Prato and Grisvard (1982), Engel and Nagel (2001), Hille and Philips (1975), Nagel and Sinestrari (1994) and the references therein.

Definition 1 (Agarwal et al. 2011) Let $A$ be a linear operator with domain $D(A)$. We say that $(A, D(A))$ is a Hille-Yosida operator on $X$ if there exist $\omega \in R$ and a positive constant $M \geq 1$ such that $(\omega, \infty) \subseteq \rho(A)$ and $\sup \left\{(\lambda-\omega)^{n}\|(\lambda-A)\|^{-n}\right\} \leq M$. The infinimum of such a $\omega$ is called the type of $A$. If the constant $\omega$ can be chosen smaller than zero, $A$ is said to be of negative type.

From the Hille-Yosida theorem (Engel and Nagel 2001, Theorem II.3.8) we have the following result.

Lemma 1 Let $(A, \quad D(A))$ be a Hille-Yosida operator on $X, \quad X_{0}=\overline{D(A)}$, $D\left(A_{0}\right)=\left\{x \in D(A): A x \in X_{0}\right\}$ and $A_{0}: D\left(A_{0}\right) \subset X_{0} \rightarrow X_{0}$ be the operator defined by $A_{0} x=A x$. The operator $A_{0}$ generates a $C_{0}$ semigroup $\left(T_{0}(t)\right)_{t \geq 0}$ on $X_{0}$ with $\left\|T_{0}(t)\right\| \leq M e^{\omega t}$ for $t \geq 0$. Moreover, $\rho(A) \subset \rho\left(A_{0}\right)$ and $R\left(\lambda, A_{0}\right)=\left.R(\lambda, A)\right|_{X_{0}}$ for $\lambda \in \rho(A)$.

Let $\lambda \in \rho(A)$. we define a norm on space $X_{0}$ by

$$
\|x\|_{-1}=\left\|R\left(\lambda, A_{0}\right) x\right\|, \quad x \in X_{0} .
$$


The completion of $\left(X_{0},\|\cdot\|_{-1}\right)$ will be called the extrapolation space of $X_{0}$ associated with $A_{0}$ and will be denoted by $X_{-1}$. One can show easily that, $T_{0}(t)$ has a unique bounded linear extension $T_{-1}(t)$ to $X_{-1}$. The operator family $\left(T_{-1}(t)\right)_{t \geq 0}$ is a $C_{0}$ semigroup on $X_{-1}$, called the extrapolated semigroup of $\left(T_{0}(t)\right)_{t \geq 0}$. The domain of its generator $A_{-1}$ is equal to $A_{0}$.

The following results have been established in Amir and Maniar (1999), Agarwal et al. (2011), Nagel and Sinestrari (1994).

Lemma 2 Under the previous conditions, the following properties are verified.

(i) $D\left(A_{-1}\right)=X_{0}$ and $\left\|T_{-1}(t)\right\|_{B\left(X_{-1}\right)}=\left\|T_{0}(t)\right\|_{B\left(X_{0}\right)}$ for every $t \geq 0$;

(ii) The operator $A_{-1}: X_{0} \rightarrow X_{-1}$ is the unique continuous extension of $A_{0}: D\left(A_{0}\right) \subset\left(X_{0},\|\cdot\|\right) \rightarrow\left(X_{-1},\|\cdot\|_{-1}\right)$ and $\lambda-A_{-1}$ is an isometry from $\left(X_{0},\|\cdot\|\right)$ to $\left(X_{-1},\|\cdot\|_{-1}\right)$;

(iii) If $\lambda \in \rho\left(A_{0}\right)$, then $\left(\lambda-A_{-1}\right)^{-1}$ exists and $\left(\lambda-A_{-1}\right)^{-1} \in B\left(X_{-1}\right)$. In particular, $\lambda \in \rho\left(A_{-1}\right)$ and $\left.R\left(\lambda, A_{-1}\right)\right|_{X_{0}}=R\left(\lambda, A_{0}\right)$;

(iv) The space $X_{0}=\overline{D(A)}$ is dense in $\left(X_{-1},\|\cdot\|_{-1}\right)$. Thus, the extrapolation space $X_{-1}$ is also the completion of $\left(X,\|\cdot\|_{-1}\right)$ and $X \hookrightarrow X_{-1}$. Moreover, $A_{-1}$ is an extension of $A$ to $X_{-1}$. In particular, if $\lambda \in \rho(A)$, then $\left.R\left(\lambda, A_{-1}\right)\right|_{X}=R(\lambda, A)$ and $R\left(\lambda, A_{-1}\right)=D(A)$.

Lemma $3 f \in C_{b}(R, X)$ and $(A, D(A))$ be a Hille-Yosida operator of negative $\omega$-type. Then the following properties are valid.

(i) $\int_{-\infty}^{t} T_{-1}(t-s) f(s) d s \in X_{0}$, for every $t \in R$;

(ii) $\left\|\int_{-\infty}^{t} T_{-1}(t-s) f(s) d s\right\| \leq M e^{\omega t} \int_{-\infty}^{t} e^{-\omega s}\|f(s)\| d s$ where $M>0$ is independent of t and f;

(iii) The function $\Gamma(f)(t)=\int_{-\infty}^{t} T_{-1}(t-s) f(s) d s \in X_{0}$ is continuous, where the operator $\Gamma: C_{b}(R, X) \rightarrow C_{b}\left(R, X_{0}\right)$.

Now, we recall a useful compactness criterion.

Let $h: R \rightarrow R$ be a continuous function such that $h(t) \geq 1$ for all $t \in R$, and $h(t) \rightarrow \infty$ as $|t| \rightarrow \infty$. We consider the space

$$
C_{h}(X)=\left\{u \in C(R, X): \lim _{|t| \rightarrow \infty} \frac{u(t)}{h(t)}=0\right\}
$$

endowed with the norm

$$
\|u\|_{h}=\sup _{t \in R} \frac{\|u(t)\|}{h(t)} .
$$

Lemma 4 (Henríquez and Lizama 2009) A subset $K \subseteq C_{h}(X)$ is a relatively compact set if it verifies the following conditions:

(i) The set $K(t)=\{u(t): u \in K\}$ is relatively compact in $X$ for each $t \in R$;

(ii) The set $K$ is equicontinuous;

(iii) For each $\varepsilon>0$ there exists $L>0$ such that $\|u(t)\| \leq \varepsilon h(t)$ for all $u \in K$ and all $|t|>L$. 
Remark 1 (Henríquez and Lizama 2009) It is clear that $C_{h}(X)$ is a Banach space isometrically isomorphic with the space $C_{0}(R, X)$ consisting of functions that vanish at infinity.

Also, we recall some notations about Stepanov bounded functions and anti-periodic functions.

Definition 2 (Pankov 1990) The Bochner transform $f^{b}(t, s), t \in R, s \in[0,1]$, of a function $f(t)$ on $R$, with values in $X$, is defined by

$$
f^{b}(t, s):=f(t+s)
$$

Definition 3 (Pankov 1990) Let $p \in[1, \infty)$. The space $B S^{p}(X)$ of all Stepanov bounded functions, with the exponent $p$, consists of all measurable functions $f$ on $R$ with values in $X$ such that $f^{b} \in L^{\infty}\left(R, L^{p}(0,1 ; X)\right)$. This is a Banach space with the norm

$$
\|f\|_{S^{p}}=\left\|f^{b}\right\|_{L^{\infty}\left(R, L^{p}\right)}=\sup _{t \in R}\left(\int_{t}^{t+1}\|f(\tau)\|^{p} d \tau\right)^{1 / p} .
$$

Definition 4 (Aizicovici et al. 2001) A function $f \in C_{b}(R, X)$ is called anti-periodic provided that

$$
f(t+T)=-f(t), \quad \forall t \in R .
$$

Denote by $P_{T A}(R, X)$ the set of all anti-periodic functions.

Lemma 5 (N'Guérékata and Valmorin 2012) Let $f_{n} \in P_{T A}(R, X)$, such that $f_{n} \rightarrow f$ uniformly on $R$. Then $f \in P_{T A}(R, X)$.

Lemma 6 (N'Guérékata and Valmorin 2012) The $P_{T A}(R, X)$ is a Banach space equipped with the supnorm.

Lemma 7 (N'Guérékata and Valmorin 2012) If $f \in C^{1}(R, X)$ is anti-periodic, then $f^{\prime} \in P_{T A}(R, X)$.

Definition 5 A continuous function $f$ is said to be anti-periodic differentiable if $f \in P_{T A}(R, X)$ and $f^{\prime} \in P_{T A}(R, X)$.

Denote by $P_{T A}^{\prime}(R, X)$ the set of all such functions.

Lemma 8 (Liu et al. 2015) $P_{T A}^{\prime}(R, X)$ is a Banach space with the supremum norm given by

$$
\|f\|_{P_{T A}^{\prime}(R, X)}=\sup _{t \in R}\left(\|f(t)\|+\left\|f^{\prime}(t)\right\|\right) .
$$

Definition 6 (Amir and Maniar 2000) Let $(A, D(A))$ be a Hille-Yosida operator of negative $\omega$-type. A function $u(t): R \rightarrow X$ satisfying the equation

$$
u(t)=T_{0}(t-s) u(s)+\int_{s}^{t} T_{-1}(t-\tau) f(\tau, u(\tau)) d \tau
$$


for all $t \geq s>-\infty$ is called a mild solution of semilinear differential equation

$$
u^{\prime}(t)=A u(t)+f(t, u(t)), t \in R .
$$

We give the famous Schauder's fixed point theorem as follows:

Lemma 9 (Smart 1980) Let D be a nonempty, closed, bounded, convex subset of a Banach space X. Let $F: D \rightarrow D$ be a continuous and compact operator, then the operator equation $F u=u$ has a fixed point in $D$.

\section{Main results and proofs}

In this section, we study the existence of anti-periodic (or anti-periodic differentiable) mild solutions of Eq. (1). The following are the main results of this work.

First, we list some assumptions.

$\left(H_{1}\right) \quad$ The function $f: R \times X_{0} \rightarrow X$ is continuous and $f(t+T,-u)=-f(t, u)$ for all $t \in R, u \in X_{0}$

$\left(H_{2}\right)$ The function $f: R \times X_{0} \rightarrow X$ satisfies the condition:

$$
\|f(t, x)-f(t, y)\| \leq L(t)\|x-y\|
$$

for all $t \in R, x, y \in X_{0}$, where $L(t) \in B S^{p}(R)$.The following theorem is needed to establish our next results.

Theorem 1 Let $(A, D(A))$ be a Hille-Yosida operator of negative $\omega$-type and $f$ satisfy the condition $\left(H_{1}\right)$. The $\Gamma: C_{b}(R, X) \rightarrow C_{b}\left(R, X_{0}\right)$ is a linear operator and $\Gamma u(t)$ is defined by

$$
\Gamma u(t)=\int_{-\infty}^{t} T_{-1}(t-s) f(s, u(s)) d s
$$

for every $t \in R$.

$$
\text { If } u \in P_{T A}\left(R, X_{0}\right) \text {, then } \Gamma u(t) \in P_{T A}\left(R, X_{0}\right) \text {. }
$$

Proof Firstly, it is easily to see that

$$
\begin{aligned}
\|\Gamma u(t)\| & \leq \int_{-\infty}^{t}\left\|T_{-1}(t-s) f(s, u(s))\right\| d s \\
& \leq \int_{-\infty}^{t} M e^{\omega(t-s)}\|f(s, u(s))\| d s \\
& \leq \frac{M}{-\omega}\|f\| .
\end{aligned}
$$


Thus $\Gamma$ is well defined and $\Gamma u$ is bounded.

Secondly, for any $t \in R, \quad h \in R$ is small enough

$$
\begin{aligned}
\|\Gamma u(t+h)-\Gamma u(t)\| & =\left\|\int_{-\infty}^{t+h} T_{-1}(t+h-s) f(s, u(s)) d s-\int_{-\infty}^{t} T_{-1}(t-s) f(s, u(s)) d s\right\| \\
& =\left\|\int_{-\infty}^{t} T_{-1}(t-s)[f(s+h, u(s+h))-f(s, u(s))] d s\right\| \\
& \leq M e^{\omega t} \int_{-\infty}^{t} e^{-\omega s}\|f(s+h, u(s+h))-f(s, u(s))\| d s \\
& \leq \frac{M}{-\omega}\|f(s+h, u(s+h))-f(s, u(s))\| .
\end{aligned}
$$

Thus, $\|\Gamma u(t+h)-\Gamma u(t)\| \rightarrow 0$ as $h \rightarrow 0$, which proves that $\Gamma u$ is continuous.

Finally, It follows from $\left(H_{1}\right)$ that for any $u \in P_{T A}\left(R, X_{0}\right)$ and for each $t \in R$

$$
\begin{aligned}
\Gamma u(t+T) & =\int_{-\infty}^{t+T} T_{-1}(t+T-s) f(s, u(s)) d s \\
& =\int_{-\infty}^{t} T_{-1}(t-s) f(s+T, u(s+T)) d s \\
& =-\int_{-\infty}^{t} T_{-1}(t-s) f(s, u(s)) d s \\
& =-\Gamma u(t) .
\end{aligned}
$$

Therefore, $\Gamma u$ is anti-periodic. The proof is complete.

Theorem 2 Let $(A, D(A))$ be a Hille-Yosida operator of negative $\omega$-type and $f$ satisfy the conditions $\left(H_{1}\right)$ and $\left(H_{2}\right)$. Then Eq. (1) has a unique anti-periodic mild solution provided that

$$
\left(\frac{e^{\omega q}-1}{\omega q}\right)^{\frac{1}{q}} \frac{M}{1-e^{\omega}}\|L\|_{S^{P}}<1, \quad \frac{1}{p}+\frac{1}{q}=1 .
$$

Proof Define a operator $\Gamma$ as in Theorem 1 on $P_{T A}\left(R, X_{0}\right)$ by

$$
\Gamma u(t)=\int_{-\infty}^{t} T_{-1}(t-s) f(s, u(s)) d s
$$

for every $t \in R$. By Theorem 1 , the operator $\Gamma$ is well defined and maps $P_{T A}\left(R, X_{0}\right)$ into itself.

Next, we prove that the operator $\Gamma$ has a unique fixed point in $P_{T A}\left(R, X_{0}\right)$.

Let $u, v \in P_{T A}\left(R, X_{0}\right)$, then 


$$
\begin{aligned}
& \|\Gamma u(t)-\Gamma v(t)\|=\left\|\int_{-\infty}^{t} T_{-1}(t-s)[f(s, u(s))-f(s, v(s))] d s\right\| \\
& \leq M \int_{-\infty}^{t} e^{\omega(t-s)}\|f(s, u(s))-f(s, v(s))\| d s \\
& \leq M \int_{0}^{\infty} e^{\omega s} L(t-s)\|u-v\| d s \\
& \leq M\|u-v\| \sum_{k \geq 0} \int_{k}^{k+1} e^{\omega s} L(t-s) d s \\
& \leq M\|u-v\| \sum_{k \geq 0}\left(\int_{k}^{k+1} e^{\omega q s} d s\right)^{\frac{1}{q}}\left(\int_{k}^{k+1} L^{p}(t-s) d s\right)^{\frac{1}{p}} \\
& \leq M\|L\|_{S^{P}}\|u-v\| \sum_{k \geq 0}\left(\int_{k}^{k+1} e^{\omega q s} d s\right)^{\frac{1}{q}} \\
& \leq\left(\frac{e^{\omega q}-1}{\omega q}\right)^{\frac{1}{q}} \frac{M}{1-e^{\omega}}\|L\|_{S^{P}}\|u-v\| .
\end{aligned}
$$

For $\left(\frac{e^{\omega q}-1}{\omega q}\right)^{\frac{1}{q}} \frac{M}{1-e^{\omega}}\|L\|_{S^{P}}<1$, it follows from the Banach contraction mapping principle that $\Gamma$ admits a unique fixed point $u \in P_{T A}\left(R, X_{0}\right)$.

Moreover, one can see easily that $u(t)$ satisfies the variation of constants formula

$$
u(t)=T_{0}(t-s) u(s)+\int_{s}^{t} T_{-1}(t-\tau) f(\tau, u(\tau)) d \tau
$$

that is $u(t)$ is a mild solution of Eq. (1). The proof is complete.

Theorem 3 Let $(A, D(A))$ be a Hille-Yosida operator of negative $\omega$-type and $f$ satisfy the conditions $\left(H_{1}\right)$ and $\left(H_{2}\right)$. If $L(t) \in B S^{1}(R)$, then Eq. (1) has a unique anti-periodic mild solution provided that $0<\frac{M}{1-e^{\omega}}\|L\|_{S^{1}}<1$.

Proof Define a operator $\Gamma$ as in Theorem 1 on $P_{T A}\left(R, X_{0}\right)$ by

$$
\Gamma u(t)=\int_{-\infty}^{t} T_{-1}(t-s) f(s, u(s)) d s
$$

for every $t \in R$. By Theorem 1 , the operator $\Gamma$ is well defined and maps $P_{T A}\left(R, X_{0}\right)$ into itself.

Next, we prove that the operator $\Gamma$ has a unique fixed point in $P_{T A}\left(R, X_{0}\right)$.

Let $u, v \in P_{T A}\left(R, X_{0}\right)$, then 


$$
\begin{aligned}
\|\Gamma u(t)-\Gamma v(t)\| & =\left\|\int_{-\infty}^{t} T_{-1}(t-s)[f(s, u(s))-f(s, v(s))] d s\right\| \\
& \leq M \int_{-\infty}^{t} e^{\omega(t-s)}\|f(s, u(s))-f(s, v(s))\| d s \\
& \leq M \int_{-\infty}^{t} e^{\omega(t-s)} L(s)\|u-v\| d s \\
& \leq M\|u-v\| \sum_{k \geq 0} e^{\omega k} \int_{t-k-1}^{t-k} L(s) d s \\
& \leq M\|u-v\| \sum_{k \geq 0} e^{\omega k}\|L\|_{S^{1}} \\
& \leq \frac{M}{1-e^{\omega}}\|L\|_{S^{1}}\|u-v\| .
\end{aligned}
$$

For $0<\frac{M}{1-e^{\omega}}\|L\|_{S^{1}}<1$, it follows from the Banach contraction mapping principle that $\Gamma$ admits a unique fixed point $u \in P_{T A}\left(R, X_{0}\right)$. The proof is complete.

Let $L(\cdot) \equiv L$, then the following result is now immediate.

Theorem 4 Let $(A, D(A))$ be a Hille-Yosida operator of negative $\omega$-type. The function $f$ satisfies the condition $\left(H_{1}\right)$ and the Lipschitz condition

$$
\|f(t, x)-f(t, y)\| \leq L\|x-y\|
$$

or all $t \in R, x, y \in X_{0}$, where $L>0$ is a constant. If $\frac{M L}{-\omega}<1$ and $\omega<0$, then the Eq. (1) has a unique anti-periodic mild solution.

Proof Similar as the proof of Theorem 3, the proof is omitted.

Theorem 5 Let $(A, D(A))$ be a Hille-Yosida operator of negative $\omega$-type. The function $f \in C_{b}^{1}\left(R, X_{0}\right)$ satisfies the condition $\left(H_{1}\right)$ and

$$
\|f(t, x)-f(t, y)\|_{C_{b}^{1}\left(R, X_{0}\right)} \leq L(t)\|x-y\|_{C_{b}^{1}\left(R, X_{0}\right)}
$$

for all $t \in R, x, y \in C_{b}^{1}\left(R, X_{0}\right)$, where $L(t) \in B S^{P}(R)$. If

$$
\left(\frac{e^{\omega q}-1}{\omega q}\right)^{\frac{1}{q}} \frac{M}{1-e^{\omega}}\|L\|_{S^{P}}<1, \quad \frac{1}{p}+\frac{1}{q}=1,
$$

then the Eq. (1) has a unique anti-periodic differentiable mild solution.

Proof Consider the nonlinear operator $V: P_{T A}^{\prime}\left(R, X_{0}\right) \rightarrow C_{b}\left(R, X_{0}\right)$ given by

$$
(V u)(t)=\int_{-\infty}^{t} T_{-1}(t-s) f(s, u(s)) d s, \quad t \in R .
$$

Firstly, similar as the proof of Theorem $1, V \in C_{b}\left(R, X_{0}\right)$ is well defined. 
Next, we will prove that $V u,(V u)^{\prime} \in P_{T A}\left(R, X_{0}\right)$. Let $g(\cdot)=f(\cdot, u(\cdot))$, Since $f \in C^{1}\left(R, X_{0}\right)$ and satisfies condition $\left(H_{1}\right)$, by Lemma 7 , we have $g(t+T)=-g(t)$ and $g^{\prime}(t+T)=-g^{\prime}(t)$ for all $t \in R$. So

$$
\begin{aligned}
(V u)(t+T) & =\int_{-\infty}^{t+T} T_{-1}(t+T-s) g(s) d s \\
& =\int_{-\infty}^{t} T_{-1}(t-s) g(s+T) d s \\
& =-\int_{-\infty}^{t} T_{-1}(t-s) g(s) d s \\
= & -(V u)(t),
\end{aligned}
$$

we have $V u \in P_{T A}\left(R, X_{0}\right)$. Furthermore,

$$
(V u)^{\prime}(t)=\int_{-\infty}^{t} T_{-1}(t-s) g^{\prime}(s) d s, t \in R,
$$

then $(V u)^{\prime} \in P_{T A}\left(R, X_{0}\right)$.

Finally, we take $u, v \in P_{T A}^{\prime}\left(R, X_{0}\right)$, then we have

$$
\begin{aligned}
\|(V u)(t)-(V v)(t)\|_{P_{T A}^{\prime}\left(R, X_{0}\right)} & =\left\|\int_{-\infty}^{t} T_{-1}(t-s)[f(s, u(s))-f(s, v(s))] d s\right\|_{P_{T A}^{\prime}\left(R, X_{0}\right)} \\
& \leq \int_{-\infty}^{t}\left\|T_{-1}(t-s)\right\| L(s)\|u(s)-v(s)\|_{P_{T A}^{\prime}\left(R, X_{0}\right)} d s \\
& \leq M\|u-v\|_{P_{T A}^{\prime}\left(R, X_{0}\right)} \sum_{k \geq 0} \int_{k}^{k+1} e^{\omega s} L(t-s) d s \\
& \leq\left(\frac{e^{\omega q}-1}{\omega q}\right)^{\frac{1}{q}} \frac{M}{1-e^{\omega}}\|L\|_{S^{P}}\|u-v\|_{P_{T A}^{\prime}\left(R, X_{0}\right)}
\end{aligned}
$$

which prove that $V$ is a contraction. Hence, by using Banach contraction mapping principle that $V$ admits a unique fixed point $u \in P_{T A}^{\prime}\left(R, X_{0}\right)$. The proof is complete.

We next study the existence of anti-periodic mild solutions of Eq. (1) when the function $\mathrm{f}$ is not Lipschitz continuous. To abridge the text, We assume that $f: R \times X_{0} \rightarrow X$ satisfies the following conditions:

$\left(A_{1}\right)$ There is a continuous nondecreasing function $W: R^{+} \rightarrow R^{+}$, such that

$$
\|f(t, x)\| \leq W(\|x\|)
$$

for all $t \in R, x \in X_{0}$;

$\left(A_{2}\right)$ For each $\kappa \geq 0$, let $\beta(\kappa)=\int_{-\infty}^{t} e^{\omega(t-s)} W(\kappa h(s)) d s \in C_{b}(R)$ and $M \beta(\kappa) \leq r$;

$\left(A_{3}\right)$ For each $\epsilon>0$, there is a $\delta>0$, such that for every $u, v \in C_{h}\left(X_{0}\right),\|u-v\|_{h} \leq \delta$ implies

$$
\sup _{t \in R} \int_{-\infty}^{t} e^{\omega(t-s)}\|f(s, u)-f(s, v)\| d s \leq \epsilon
$$

$\left(A_{4}\right) T_{0}(t)$ is a strongly continuous $C_{0}$ semigroup. Moreover, $T_{0}(t)$ is compact. 
Theorem 6 Let $(A, D(A))$ be a Hille-Yosida operator of negative $\omega$-type. The function $f$ satisfies the conditions $\left(H_{1}\right),\left(A_{1}\right)-\left(A_{4}\right)$, then Eq. (1) has an anti-periodic mild solution.

Proof Let $D=\left\{u \in P_{T A}\left(R, X_{0}\right) \cap C_{h}\left(X_{0}\right):\|u\| \leq r\right\}$, and $D(t):=\{\Gamma u: u \in D\}$. We define the operator $\Gamma$ by

$$
\Gamma u(t)=\int_{-\infty}^{t} T_{-1}(t-s) f(s, u(s)) d s
$$

We divide the proof in several steps.

Step 1. For $u \in D$, we have that

$$
\begin{aligned}
\|\Gamma u(t)\| & =\left\|\int_{-\infty}^{t} T_{-1}(t-s) f(s, u(s)) d s\right\| \\
& \leq M \int_{-\infty}^{t} e^{\omega(t-s)}\|f(s, u(s))\| d s \\
& \leq M \int_{-\infty}^{t} e^{\omega(t-s)} W(\|u(s)\|) d s \\
& \leq M \int_{-\infty}^{t} e^{\omega(t-s)} W\left(\|u\|_{h} h(s)\right) d s \\
& \leq M \beta\left(\|u\|_{h}\right) \\
& \leq r .
\end{aligned}
$$

So $\|\Gamma u\| \leq r$. It follows from condition $\left(A_{2}\right)$ that $\Gamma: C_{h}\left(X_{0}\right) \rightarrow C_{h}\left(X_{0}\right)$.

Step 2. The map $\Gamma$ is continuous. In fact, for $\epsilon>0$, we take $\delta$ involved in condition $\left(A_{3}\right)$. If $u, v \in C_{h}\left(X_{0}\right)$ and $\|u-v\|_{h} \leq \delta$, then

$$
\begin{aligned}
\|\Gamma u(t)-\Gamma v(t)\|_{h} & =\frac{\left\|\int_{-\infty}^{t} T_{-1}(t-s)[f(s, u(s))-f(s, v(s))] d s\right\|}{h(t)} \\
& \leq \frac{M \int_{-\infty}^{t} e^{\omega(t-s)}\|f(s, u(s))-f(s, v(s))\| d s}{h(t)} \\
& \leq M \epsilon,
\end{aligned}
$$

which shows the assertion.

Step 3. We will show that $\Gamma$ is a compact operator.

We will prove that $D(t):=\{\Gamma u: u \in D\}$ is a relatively compact subset of $X_{0}$ for each $t \in R$.

For each $t \in R, 0<\varepsilon<1$, define

$$
\begin{aligned}
\Gamma_{\varepsilon} u & =\int_{-\infty}^{t-\varepsilon} T_{-1}(t-s) f(s, u(s)) d s \\
& =T_{0}(\varepsilon) \int_{-\infty}^{t-\varepsilon} T_{-1}(t-\varepsilon-s) f(s, u(s)) d s \\
& =T_{0}(\varepsilon) \Gamma u(t-\varepsilon) .
\end{aligned}
$$

Since $\{\Gamma u(t-\varepsilon)\}$ is bounded and $T_{0}(\varepsilon)$ is compact, $\left\{\Gamma_{\varepsilon} u, u \in D\right\}$ is a relatively compact subset of $X_{0}$, then 


$$
\begin{aligned}
\left\|\Gamma u-\Gamma_{\varepsilon} u\right\| & =\left\|\int_{t-\varepsilon}^{t} T_{-1}(t-s) f(s, u(s)) d s\right\| \\
& \leq \int_{t-\varepsilon}^{t} M e^{\omega(t-s)}\|f(s, u(s))\| d s \\
& \leq M \int_{t-\varepsilon}^{t} e^{\omega(t-s)} W(\|u(s)\|) d s \\
& \leq M \int_{t-\varepsilon}^{t} e^{\omega(t-s)} W\left(\|u(s)\|_{h} h(s)\right) d s . \\
& \leq M W\left(\|u(s)\|_{h} h(s)\right) \varepsilon .
\end{aligned}
$$

So $\left\|\Gamma u-\Gamma_{\varepsilon} u\right\|_{h} \rightarrow 0$, as $\varepsilon \rightarrow 0$.

Thus, $D(t):=\{\Gamma u: u \in D\}$ is a relatively compact subset of $X_{0}$ for each $t \in R$.

Next,we will show that the set $D$ is equicontinuous.

In fact, proceeding as above, for $t_{1}<t_{2}, t_{1}, t_{2} \in R$, we can decompose

$$
\begin{aligned}
\Gamma u\left(t_{2}\right)-\Gamma u\left(t_{1}\right)= & \int_{-\infty}^{t_{2}} T_{-1}\left(t_{2}-s\right) f(s, u(s)) d s-\int_{-\infty}^{t_{1}} T_{-1}\left(t_{1}-s\right) f(s, u(s)) d s \\
= & \int_{-\infty}^{t_{1}-\gamma}\left(T_{-1}\left(t_{2}-s\right)-T_{-1}\left(t_{1}-s\right)\right) f(s, u(s)) d s \\
& +\int_{t_{1}-\gamma}^{t_{1}}\left(T_{-1}\left(t_{2}-s\right)-T_{-1}\left(t_{1}-s\right)\right) f(s, u(s)) d s \\
& +\int_{t_{1}}^{t_{2}} T_{-1}\left(t_{2}-s\right) f(s, u(s)) d s .
\end{aligned}
$$

For each $\epsilon>0$,

$$
\begin{aligned}
& \left\|\int_{-\infty}^{t_{1}-\gamma}\left(T_{-1}\left(t_{2}-s\right)-T_{-1}\left(t_{1}-s\right)\right) f(s, u(s)) d s\right\| \\
& \quad=\left\|\left(T_{0}\left(t_{2}-t_{1}+\gamma\right)-T_{0}(\gamma)\right) \int_{-\infty}^{t_{1}-\gamma} T_{-1}\left(t_{1}-\gamma-s\right) f(s, u(s)) d s\right\| \\
& \quad \leq\left\|T_{0}\left(t_{2}-t_{1}+\gamma\right)-T_{0}(\gamma)\right\|\left\|\int_{-\infty}^{t_{1}-\gamma} T_{-1}\left(t_{1}-\gamma-s\right) f(s, u(s)) d s\right\| \\
& \quad \leq\left\|T_{0}\left(t_{2}-t_{1}+\gamma\right)-T_{0}(\gamma)\right\| \int_{-\infty}^{t_{1}-\gamma} M e^{\omega\left(t_{1}-\gamma-s\right)} W\left(\|u\|_{h} h(s)\right) d s
\end{aligned}
$$

From step 1, we get $\left\|\int_{-\infty}^{t_{1}-\gamma}\left(T_{-1}\left(t_{2}-s\right)-T_{-1}\left(t_{1}-s\right)\right) f(s, u(s)) d s\right\| \rightarrow 0$ as $t_{2}-t_{1} \rightarrow 0$. Moreover, similarly estimates as proof of $\left\|\Gamma u-\Gamma_{\varepsilon} u\right\|$, when $t_{2}-t_{1} \rightarrow 0$, we can prove that

$$
\left\|\int_{t_{1}-\gamma}^{t_{1}}\left(T_{-1}\left(t_{2}-s\right)-T_{-1}\left(t_{1}-s\right)\right) f(s, u(s)) d s\right\| \rightarrow 0
$$

and

$$
\left\|\int_{t_{1}}^{t_{2}} T_{-1}\left(t_{2}-s\right) f(s, u(s)) d s\right\| \rightarrow 0 .
$$

Combining these estimates, we get 


$$
\left\|\Gamma u\left(t_{2}\right)-\Gamma u\left(t_{1}\right)\right\| \rightarrow 0
$$

as $t_{2}-t_{1} \rightarrow 0$ and independent of $u \in D$.

Finally, applying condition $\left(A_{2}\right)$, we can show that

$$
\frac{\|\Gamma u\|}{h(t)} \leq \frac{M}{h(t)} \int_{-\infty}^{t} e^{\omega(t-s)} W(r h(s)) d s \rightarrow 0, \quad|t| \rightarrow \infty
$$

and this convergence is independent of $u \in D$.

Hence $D$ satisfies conditions (i)-(iii) of Lemma 4 , so $D$ is a relatively compact set in $C_{h}\left(X_{0}\right)$. It follows from the proof of steps $1-3$ that $\Gamma$ is a compact operator.

Step4. Applying with Theorem 1, we obtain that $\Gamma\left(P_{T A}\left(R, X_{0}\right)\right) \subseteq P_{T A}\left(R, X_{0}\right)$. Consequently, combining with step 1 and step 2 we infer that $\Gamma\left(P_{T A}\left(R, X_{0}\right) \cap C_{h}\left(X_{0}\right)\right) \subseteq P_{T A}\left(R, X_{0}\right) \cap C_{h}\left(X_{0}\right)$, and also

$$
\Gamma\left(\overline{P_{T A}\left(R, X_{0}\right) \cap C_{h}\left(X_{0}\right)}{ }^{h}\right) \subseteq \overline{\Gamma\left(P_{T A}\left(R, X_{0}\right) \cap C_{h}\left(X_{0}\right)\right)}{ }^{h} \subseteq{\overline{P_{T A}\left(R, X_{0}\right) \cap C_{h}\left(X_{0}\right)}}^{h} .
$$

where $\bar{D}^{h}$ denotes the closure of $D$ in $C_{h}\left(X_{0}\right)$. Applying the Lemma 9 (Schauder's Fixed Point Theorem), we deduce that $\Gamma$ has a fixed point $u \in \overline{P_{T A}\left(R, X_{0}\right) \cap C_{h}\left(X_{0}\right)}{ }^{h}$.

Step 5. we prove that $u \in P_{T A}\left(R, X_{0}\right)$.

Let $\left(u_{n}\right)_{n}$ be a sequence in $P_{T A}\left(R, X_{0}\right) \cap C_{h}\left(X_{0}\right)$ that converges to $u$ for the topology in $C_{h}\left(X_{0}\right)$. It follows from condition $\left(A_{3}\right)$ that $\Gamma u_{n} \rightarrow \Gamma u$ as $n \rightarrow \infty$, uniformly on $R$. This implies that $u \in P_{T A}\left(R, X_{0}\right)$, which completes the proof.

Remark 2 The assumption $\left(A_{3}\right)$ of Theorem 6 is fulfilled in the following situation:

$$
\|f(t, h(t) x)-f(t, h(t) y)\| \leq W(\|x-y\|)
$$

for all $t \in R, x, y \in X_{0}$.

In fact, we use the same notations as in Theorem 6.

$$
\begin{aligned}
\|\Gamma u(t)-\Gamma v(t)\| & =\left\|\int_{-\infty}^{t} T_{-1}(t-s)[f(s, u(s))-f(s, v(s))] d s\right\| \\
& \leq M \int_{-\infty}^{t} e^{\omega(t-s)} W\left(\frac{\|u(s)-v(s)\|}{h(s)}\right) d s \\
& \leq \frac{M}{-\omega} W\left(\|u-v\|_{h}\right) .
\end{aligned}
$$

Since $W$ is continuous, the above estimate shows that $\left(A_{3}\right)$ hold, the remains of proof is essentially the same of Theorem 6.

\section{An example}

In this section we give an example to illustrate the above results. Consider the following partial differential equation:

$$
\partial_{t} u(t, x)=\partial_{x}^{2} u(t, x)-\omega u(t, x)+F(t, u(t, x)), \quad t \in R, x \in[0, \pi], \quad \omega<0,
$$

with boundary initial conditions 


$$
u(t, 0)=u(t, \pi)=0, \quad t \in R .
$$

Let $X=C([0, \pi], R)$ and the operator $A$ be defined on $X$ by $A u=u^{\prime \prime}-\omega u$, with domain

$$
D(A)=\left\{u \in X: u^{\prime \prime} \in X, u(0)=u(\pi)=0\right\} .
$$

It is well known that $A$ is a Hille-Yosida operator of type- $\omega$ with domain nondense (Prato and Sinestrari 1987). The above partial differential equation can be rewritten as an abstract system of the Eq. (1), where $u(t)(s)=u(t, s)$.

Let us consider the nonlinearity $F(t, x)(s)=\beta b(t) \sin (x(s))$ for all $x \in X$ and $s \in[0, \pi]$, $t \in R$, where $b(t)$ is a bounded periodic function with period $T$, thus we have

$$
F(t+T,-x)(s)=\beta b(t+T) \sin (-x(s))=-\beta b(t) \sin (x(s))=-F(t, x)(s)
$$

and

$$
\|F(t, x)-F(t, y)\|=\|\beta b(t) \sin (x(s))-\beta b(t) \sin (y(s))\| \leq|\beta\|b(t) \mid\| x-y \| .
$$

If $\int_{-\infty}^{t} e^{\omega(t-s)} b(s) d s<\frac{1}{|\beta| M}$, where $\omega<0$. Then the Eq. (2) with boundary initial conditions (3) has a unique anti-periodic mild solutions.

\section{Conclusions}

This paper is concerned with the semilinear differential equation $u^{\prime}(t)=A u(t)+f(t, u(t))$ with nondense domain. Under some suitable conditions, we establish the existence of anti-periodic (or anti-periodic differentiable) mild solutions to the semilinear differential equation. To the best of our knowledge, it is the first time to deal with this problem. Moreover, the method of this paper can be applied to many other differential equations, such as impulsive differential equations, neutral functional differential equations, fractional differential equations and so on.

\section{Authors' contributions}

All authors contributed equally to the manuscript. Both authors read and approved the final manuscript.

\section{Author details}

1 Department of Mathematics and Physics, Zhengzhou University of Aeronautics, Zhengzhou 450015, People's Republic of China. ${ }^{2}$ Collaborative Innovation Center for Aviation Economy Development of Henan Province, Zhengzhou University of Aeronautics, Zhengzhou 450015, People's Republic of China.

\section{Acknowledgements}

The authors would like to thank the referee for the valuable comments and suggestions. This work is supported by the National Natural Science Foundation of China (11226337), National Natural Science Foundation of China (51401182) and the Science and Technology Research Projects of Henan Education Committee(16A110024).

\section{Competing interests}

The authors declare that they have no competing interests.

Received: 10 December 2015 Accepted: 10 May 2016

Published online: 10 June 2016

\section{References}

Abdurahman A, Jiang H (2015) The existence and stability of the anti-periodic solution for delayed Cohen-Grossberg neural networks with impulsive effects. Neurocomputing 149:22-28

Aftabizadeh AR, Pavel NH, Huang YK (1994) Anti-periodic oscillations of some second-order differential equations and optimal control problems. J Comput Appl Math 52(1):3-21

Agarwal RP, Cuevas C, Soto H, El-Gebeily M (2011) Asymptotic periodicity for some evolution equations in Banach spaces. Nonlinear Anal 74(5):1769-1798

Aizicovici S, McKibben M, Reich S (2001) Anti-periodic solutions to nonmonotone evolution equations with discontinuous nonlinearities. Nonlinear Anal 43(2):233-251 
Al-Islam NS, Alsulami SM, Diagana T (2012) Existence of weighted pseudo anti-periodic solutions to some non-autonomous differential equations. Appl Math Comput 218(11):6536-6548

Amir B, Maniar L (1999) Composition of pseudo-almost periodic functions and Cauchy problems with operator of non dense domain. Ann Math Blaise Pascal 6(1):1-11

Amir B, Maniar L (2000) Asymptotic behavior of hyperbolic Cauchy problems for Hille-Yosida operators with an application to retarded differential equations. Quaest Math 23(3):343-357

Cao J, Yang Q, Huang Z (2012) Existence of anti-periodic mild solutions for a class of semilinear fractional differential equations. Commun Nonlinear Sci Numer Simul 17(1):277-283

Chadli O, Ansari QH, Yao JC (2016) Mixed equilibrium problems and anti-periodic solutions for nonlinear evolution equations. J Optim Theory Appl 168(2):410-440

Chen YQ, Nieto JJ, O'Regan D (2007) Anti-periodic solutions for full nonlinear first-order differential equations. Math Comput Model 46(9):1183-1190

Da Prato G, Grisvard P (1982) On extrapolation spaces. Rend Accad Naz Lincei 72:330-332

Da Prato G, Sinestrari E (1987) Differential operators with nondense domain. Ann Sc Norm Super Pisa 14(2):285-344

Engel KJ, Nagel R (2001) One-parameter semigroups for linear evolution equations. Graduate texts in mathematics, vol 194. Springer, Berlin

Haraux A (1989) Anti-periodic solutions of some nonlinear evolution equations. Manuscr Math 63(4):479-505

Henríquez H, Lizama C (2009) Compact almost automorphic solutions to integral equations with infinite delay. Nonlinear Anal 71(12):6029-6037

Hille E, Philips RS (1975) Functional analysis and semigroup. American Mathematical Society, Providence, R

Liu JH, Cheng SH, Zhang LT (2015) Anti-periodic mild solutions to semilinear fractional differential equations. J Appl Math Comput 48(1-2):381-393

Liu JH, Song XQ, Zhang LT (2015) Existence of anti-periodic mild solutions to semilinear nonautonomous evolution equations. J Math Anal Appl 425(1):295-306

Nagel R, Sinestrari E (1994) Inhomegeneous volterra integrodifferential equations for Hille-Yosida operators, Marcel Dekker. Lect Notes Pure Appl Math 150:51-70

N'Guérékata GM, Valmorin V (2012) Antiperiodic solutions of semilinear integrodifferential equations in Banach spaces. Appl Math Comput 218(22):11118-11124

Okochi H (1990) On the existence of anti-periodic solutions to a nonlinear evolution equation associated with odd subdifferential operators. J Funct Anal 91(2):246-258

Pankov A (1990) Bounded and almost periodic solutions of nonlinear operator differential equations. Kluwer, Dordrecht Smart DR (1980) Fixed point theorems. Cambridge University Press, London

Wang R, Chen D (2013) Anti-periodic problems for semilinear partial neutral evolution equations. Electron J Qual Theory Differ Equ 16:1-16

Xu C (2016) Existence and exponential stability of anti-periodic solutions in cellular neural networks with time-varying delays and impulsive effects. Electron J Diff Equ 2016(02):1-14

Yang XJ, Srivastava HM (2015) An asymptotic perturbation solution for a linear oscillator of free damped vibrations in fractal medium described by local fractional derivatives. Commun Nonlinear Sci Numer Simul 29(1):499-504

\section{Submit your manuscript to a SpringerOpen ${ }^{\circ}$ journal and benefit from:}

- Convenient online submission

Rigorous peer review

- Immediate publication on acceptance

- Open access: articles freely available online

- High visibility within the field

- Retaining the copyright to your article

Submit your next manuscript at $>$ springeropen.com 